\title{
Dissipation-induced stationary entanglement in dipole-dipole interacting atomic samples
}

\author{
S. Nicolosi, ${ }^{1}$ A. Napoli, ${ }^{1}$ A. Messina, ${ }^{1, *}$ and F. Petruccione ${ }^{2}$ \\ ${ }^{1}$ INFM, MIUR, and Dipartimento di Scienze Fisiche ed Astronomiche, Università di Palermo, Via Archirafi 36, 90123 Palermo, Italy \\ ${ }^{2}$ School of Pure and Applied Physics University of KwaZulu-Natal Durban, 4041 Durban, South Africa, \\ and Istituto Italiano per gli Studi Filosofici, Via Monte di Dio 14, I-80132 Napoli, Italy
}

(Received 26 February 2004; published 24 August 2004)

\begin{abstract}
The dynamics of two two-level dipole-dipole interacting atoms coupled to a common electro-magnetic bath and closely located inside a lossy cavity, is reported. Initially injecting only one excitation in the two-atom cavity system, loss mechanisms asymptotically drive the matter sample toward a stationary maximally entangled state. The role played by the closeness of the two atoms, with respect to such a cooperative behavior, is carefully discussed. Stationary radiation trapping effects are found and transparently interpreted.
\end{abstract}

DOI: 10.1103/PhysRevA.70.022511

PACS number(s): 31.15.Ar, 03.65.Yz, 03.67.Mn, 42.50.Fx

A system prepared in an entangled state may show rather puzzling behavior and counterintuitive features. In such a state, for example, the system exhibits the astonishing property that the results of measurements on one subsystem cannot be specified independently from the parameters of the measurements on the other components. The renewed and more and more growing interest toward entanglement concept reflects the consolidated belief that unfactorizable states of multipartite system provide an unreplaceable applicative resource, for example, in the quantum computing research area [1]. However, the realization of quantum computation protocols suffers from the difficulty of isolating a quantummechanical system from its environment. Unavoidably, the system-environment interaction leads to decoherence phenomena which, as intuition suggests, are always noxious for quantum computers, since they imply the loss of the information stored in the system. This circumstance is at the origin of an intense research aimed at proposing theoretical and experimental schemes to fight or control decoherence manifestations [2-7]. Very recently, however, the possibility of environment-induced entanglement in an open quantum system has opened new intriguing perspectives [8-11]. For example, it has been shown that dissipation can be exploited to implement nearly decoherence-free quantum gates $[12,13]$, the main requirement being the existence of a decoherencefree subspace for the system under consideration [14]. Moreover, despite the widely held belief, it has been shown that transient entanglement between distant atoms can be induced by atomic spontaneous decay or by cavity losses $[9,11,15]$. It has been also demonstrated [16] that asymptotic (stationary) entangled states of two closely separated two-level atoms in free space can be created by spontaneous emission. Notwithstanding the nearness of the two atoms, dipole-dipole interaction is however neglected in Ref. [16]. In this paper we investigate the dynamics of a couple of spontaneously emitting two-level atoms confined within a bad single-mode cavity, taking into account from the very beginning their dipoledipole interaction. Our main result is that in such a condition, the matter subsystem, even experiencing a further relatively

\footnotetext{
*Electronic address: messina@fisica.unipa.it
}

faster energy loss mechanism, may as well be conditionally guided toward a stationary robust entangled state. We shall prove that this state is the antisymmetric one with respect to the exchange of the two two-level atoms provided that all the initial energy given to the system is concentrated in the matter subsystem only.

Let us suppose that two identical two-level atoms are located at $\mathbf{r}_{1}$ and $\mathbf{r}_{2}$ inside a resonant single-mode cavity. Putting $R=\left|\mathbf{r}_{1}-\mathbf{r}_{2}\right| \equiv|\mathbf{R}|$ and denoting by $\theta$ the angle between $\mathbf{R}$ and the atomic transition dipole moment $\mathbf{d}$, the Hamiltonian describing the dipole interaction between the two atoms can be written in the form $[17,18]$

$$
H_{12}=\hbar \eta\left(\sigma_{+}^{(1)} \sigma_{-}^{(2)}+\text { H.c. }\right) \text {, }
$$

where $\eta=\frac{3}{4}\left(\Gamma_{0} c^{3} / \omega_{0}^{3} R^{3}\right)\left(1-3 \cos ^{2} \theta\right)$ and $\Gamma_{0}$ is the spontaneous emission rate in free space. In Eq. (1) $\sigma_{ \pm}^{(i)}(i=1,2)$ are the Pauli operators of the $i$ th atom. Assume in addition that all the conditions are satisfied under which the interaction between each atom and the cavity field is described by a Jaynes Cummings (JC) model [19]. Under these hypotheses, the unitary time evolution of the system under scrutiny is governed by the following Hamiltonian:

$$
H_{A C}=\hbar \omega \alpha^{\dagger} \alpha+\frac{\hbar \omega_{0}}{2} \sum_{i=1}^{2} \sigma_{z}^{(i)}+\hbar \sum_{i=1}^{2}\left[\varepsilon^{(i)} \alpha \sigma_{+}^{(i)}+\text { H.c. }\right]+H_{12},
$$

where $\hbar \omega_{0}$ denotes the energy separation between the $i$ th atom $(i=1,2)$ excited $\left(|+\rangle_{i}\right)$ and ground $\left(|-\rangle_{i}\right)$ states, $\omega$ $\sim \omega_{0}$ is the frequency of the cavity mode, and $\varepsilon^{(i)}$ is the $i$ th atom-field mode coupling constant. In the above equation $\alpha$ $\left(\alpha^{\dagger}\right)$ is the annihilation (creation) operator relative to the single-mode cavity field and $\sigma_{z}^{(i)}$ the $i$ th atom inversion operator. It is easy to demonstrate that the total excitation number operator $\hat{N}=\alpha^{\dagger} \alpha+\frac{1}{2}\left(\sigma_{z}^{(1)}+\sigma_{z}^{(2)}\right)+1$ is a constant of motion. Thus, preparing the physical system at $t=0$ in a state with a well-defined number of excitations $N_{e}$, its dynamics is confined in the finite-dimensional Hilbert subspace singled out by this eigenvalue of $\hat{N}$. In a realistic situation, however, the system we are considering evolves under the action of different sources of decoherence. First, photons can leak out 
through the cavity mirrors due to the coupling of the resonator mode to the free radiation field. Moreover, the atoms confined in the resonator can spontaneously emit photons into noncavity field modes. The microscopic Hamiltonian which takes into account all these loss mechanisms can be written in the form [20]

$$
H=H_{A C}+H_{R}+H_{A R}+H_{C R},
$$

where $H_{A C}$ is given by Eq. (2),

$$
H_{R}=\hbar \sum_{\mathbf{k}, \lambda} \omega_{\mathbf{k}, \lambda}\left[c_{\mathbf{k}, \lambda}^{\dagger} c_{\mathbf{k}, \lambda}+\widetilde{c}_{\mathbf{k}, \lambda}^{\dagger} \widetilde{c}_{\mathbf{k}, \lambda}\right]
$$

is the Hamiltonian of the environment,

$$
H_{A R}=\sum_{\mathbf{k}, \lambda, i}\left[g_{\mathbf{k}, \lambda}^{(i)} \widetilde{c}_{\mathbf{k}, \lambda} \sigma_{+}^{(i)}+\text { H.c. }\right]
$$

describes the interaction between the atoms and the bath and, finally,

$$
H_{C R}=\sum_{\mathbf{k}, \lambda}\left[s_{\mathbf{k}, \lambda} c_{\mathbf{k}, \lambda} \alpha^{\dagger}+\text { H.c. }\right]
$$

takes into account the coupling between the environment and the cavity field. As usual, in Eq. (4) we have assumed that the two subsystems, the two atoms, and the single-mode cavity, see two different reservoirs in the following, both assumed at $T=0$. The boson operators relative to the atomic bath are denoted by $\left\{\widetilde{c}_{\mathbf{k}, \lambda}, \widetilde{c}_{\mathbf{k}, \lambda}^{\dagger}\right\}$, whereas $c_{\mathbf{k}, \lambda}, c_{\mathbf{k}, \lambda}^{\dagger}$ are the $(\mathbf{k}, \lambda)$ mode annihilation and creation operators, respectively, of the cavity environment. Moreover, the coupling constants $s_{\mathbf{k}, \lambda}$ are phenomenological parameters and

$$
g_{\mathbf{k}, \lambda}^{(i)}=-i\left(\frac{2 \pi \hbar \omega_{0}^{2}}{V \omega_{k}}\right)^{1 / 2} \mathbf{e}_{k \lambda} \cdot \mathbf{d} e^{i \mathbf{k} \cdot \mathbf{r}_{i}}
$$

stems from a dipole atom-field coupling [18]. In Eq. (7) $\mathbf{e}_{\mathbf{k} \lambda}$ represents the polarization vector of the thermal bath $(\mathbf{k} \lambda)$ mode of frequency $\omega_{\mathbf{k}}$ and $V$ its effective volume. In the rotating-wave and Born-Markov approximations [21,22], the reduced density operator $\rho_{A C}$ relative to the bipartite system composed by the two-atom subsystem and the single-mode cavity, evolves nonunitarily in accordance with the following quantum master equation in Lindblad form:

$$
\dot{\rho}_{A C}=-\frac{i}{\hbar}\left[H_{A C}, \rho_{A C}\right]+\mathcal{L}_{f} \rho_{A C}+\mathcal{L}_{A} \rho_{A C},
$$

where

$$
\mathcal{L}_{f} \rho_{A C}=k\left(2 \alpha \rho_{A C} \alpha^{\dagger}-\alpha^{\dagger} \rho_{A C} \alpha-\rho_{A C} \alpha^{\dagger} \alpha\right),
$$

and

$$
\begin{aligned}
\mathcal{L}_{A} \rho_{A C}= & \sum_{i=1}^{2} \Gamma\left(2 \sigma_{-}^{(i)} \rho_{A C} \sigma_{+}^{(i)}-\sigma_{+}^{(i)} \sigma_{-}^{(i)} \rho_{A C}-\rho_{A C} \sigma_{+}^{(i)} \sigma_{-}^{(i)}\right) \\
& +\Gamma_{12}\left(2 \sigma_{-}^{(1)} \rho_{A C} \sigma_{+}^{(2)}-\sigma_{+}^{(1)} \sigma_{-}^{(2)} \rho_{A C}-\rho_{A C} \sigma_{+}^{(1)} \sigma_{-}^{(2)}\right) \\
& +\Gamma_{21}\left(2 \sigma_{-}^{(2)} \rho_{A C} \sigma_{+}^{(1)}-\sigma_{+}^{(2)} \sigma_{-}^{(1)} \rho_{A C}-\rho_{A C} \sigma_{+}^{(2)} \sigma_{-}^{(1)}\right)
\end{aligned}
$$

In the above equations we have introduced $\Gamma$

$$
\begin{aligned}
& =\left(4 \pi \omega_{0}^{3}|\mathbf{d}|^{2} / 3 \hbar c^{3}\right) \text { and } \Gamma_{12} \equiv \Gamma_{21}=\Gamma f(R) \text {, where [23] }
\end{aligned}
$$

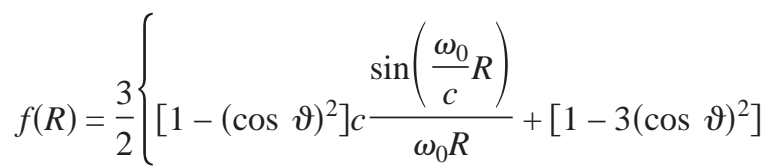

$$
\begin{aligned}
& \left.\times\left[c^{2} \frac{\sin \left(\frac{\omega_{0}}{c} R\right)}{\left(\omega_{0} R\right)^{2}}-c^{3} \frac{\sin \left(\frac{\omega_{0}}{c} R\right)}{\left(\omega_{0} R\right)^{3}}\right]\right\}
\end{aligned}
$$

with $0 \leqslant f(R) \leqslant 1$. We emphasize that $\Gamma_{12}$ measures the strength of the atom-atom cooperation induced by their coupling with a common bath. Finally, $k=\Sigma_{\mathbf{k} \lambda}\left|s_{\mathbf{k} \lambda}\right|^{2} \delta\left(\omega_{\mathbf{k}}-\omega\right)$, appearing in Eq. (9), is the cavity decay rate coefficient. Assume now that the two atoms inside the cavity are closely located, that is $c \ll R \omega_{0}$. In such a situation the cooperation between the two atoms stemming from their interaction with a common bath, is maximum, that is $f(R)=1$. Moreover, it is reasonable to put $\varepsilon^{(1)}=\varepsilon^{(2)} \equiv \varepsilon$. We are able to exactly solve Eq. (8) when this pointlike model is adopted. To this end, consider the unitary operator $U$ defined as

$$
U=\exp \left[-\frac{\pi}{4}\left(\sigma_{+}^{1} \sigma_{-}^{2}-\sigma_{-}^{1} \sigma_{+}^{2}\right)\right] .
$$

It can be shown that $[U, \hat{N}]=0$ and that, confining our attention on the Hilbert subspace correspondent to $N_{e}=0,1$, Eq. (8) can be equivalently cast in the form

$$
\begin{aligned}
\dot{\tilde{\rho}}_{A C} \equiv & U^{\dagger} \rho_{A C} U=-\frac{i}{\hbar}\left[\tilde{H}_{A C}, \tilde{\rho}_{A C}\right]+k\left(2 \alpha \widetilde{\rho}_{A C} \alpha^{\dagger}-\alpha^{\dagger} \alpha \widetilde{\rho}_{A C}\right. \\
& \left.-\widetilde{\rho}_{A C} \alpha^{\dagger} \alpha\right)+2 \Gamma\left(2 \sigma_{-}^{(1)} \tilde{\rho}_{A C} \sigma_{+}^{(1)}-\sigma_{+}^{(1)} \sigma_{-}^{(1)} \widetilde{\rho}_{A C}\right. \\
& \left.-\tilde{\rho}_{A C} \sigma_{+}^{(1)} \sigma_{-}^{(1)}\right),
\end{aligned}
$$

where $\widetilde{\rho}_{A C} \equiv U^{\dagger} \widetilde{\rho}_{A C} U$

$$
\begin{aligned}
\tilde{H}_{A C} \equiv & U^{\dagger} H_{A C} U=\hbar \omega \alpha^{\dagger} \alpha+\hbar \omega_{0} \sum_{i=1}^{2} \sigma_{z}^{(i)}+\hbar \varepsilon_{e f f}\left[\alpha \sigma_{+}^{(1)}+\text { H.c. }\right] \\
& -\frac{\hbar \eta}{2}\left(\sigma_{z}^{(2)}-\sigma_{z}^{(1)}\right),
\end{aligned}
$$

with $\varepsilon_{e f f}=\sqrt{2} \varepsilon$. It is important to notice that in the new representation, the dipole-dipole interaction $H_{12}$ given by Eq. (1) only renormalizes the atomic frequencies. The transformed Hamiltonian $\widetilde{H}_{A C}$ puts transparently into evidence that the system of two atoms cooperates in the interaction with the cavity field. In this new representation, two twolevel fictitious atoms appear, only one of them being coupled, by means of a simple JC interaction model, to the cavity field. Such a decoupling is still accomplished by $U$ when $\eta=0$. The circumstance that the atomic sample can exchange energy with the field through only one of its collective atoms, provides a natural explanation for the radiation trapping phenomena [24]. This conclusion is true under ideal conditions when neither cavity losses or atomic spontaneous decay are considered. Thus, it seems interesting to investigate whether such an energy storage mechanism survives in a more realistic situation like the one under scrutiny in this 
paper. Looking at Eq. (14) we may see also that in the transformed representation the atomic subsystem loses its energy only through the interaction of the first atom with both the cavity mode and the environment. Such a behavior stems from the fact that the other atom freely evolves being decoupled from either the cavity field and the electromagnetic modes of the thermal bath. Comparing this result with the interpretation of the energy trapping given in Refs. [24] and [25], we may immediately catch the main role played by the closeness of the two atoms in our model. It is indeed just this feature which, in the transformed representation, leads to the existence of one atom immune from spontaneous emission losses and, at the same time, decoupled from the cavity mode. Thus, to locate the atomic sample within a linear dimension much shorter than the wavelength of the cavity mode, introduces a permutational atomic symmetry which is at the origin of a collective behavior of the two atoms. As a direct consequence, the matter system may stationarily trap the initial energy even in presence of both the proposed dissipation channels. Suppose that only one excitation is initially present in the atomic subsystem, whereas the cavity is prepared in its vacuum state. From an experimental point of view it seems reasonable to think that the excitation given to the matter system is captured by the atom 1 or by the atom 2 with the same probability. On the other hand, exciting only an a priori fixed atom or preparing a quantum coherent superposition of the two states $|+\rangle_{1}|-\rangle_{2}$ and $|-\rangle_{1}|+\rangle_{2}$, could present serious practical difficulties. Stated another way, our initial condition can be reasonably expressed by $\rho_{A C}(0)$ $=\rho_{A}(0) \otimes \rho_{C}(0) \quad$ with $\quad \rho_{A}=\frac{1}{2}\left(|+\rangle_{1}|-\rangle_{2} \quad{ }_{1}\left\langle+\left.\right|_{2}\langle-|+|-\rangle_{1} \mid+\right\rangle_{21}\langle\right.$ $\left.-\left.\right|_{2}\langle+|\right)$ and $\rho_{C}(0)=|0\rangle\langle 0|| 0$,$\rangle being the vacuum Fock state of$ the cavity mode. It is evident that, under this hypothesis, at a generic time instant $t$, the density operator $\rho_{A C}$, can have nonzero matrix elements only in the Hilbert subspace generated by the following ordered set of four state vectors:

$$
\left\{|0\rangle|-\rangle_{1}|-\rangle_{2} ;|0\rangle|+\rangle_{1}|-\rangle_{2} ;|0\rangle|-\rangle_{1}|+\rangle_{2} ;|1\rangle|-\rangle_{1}|-\rangle_{2}\right\} \text {. }
$$

Taking into account that $[U, \hat{N}]=0$, the same conclusion holds for $\tilde{\rho}_{A C}$ too. This fact provides the key to solve exactly Eq. (13). One finds

$$
\tilde{\rho}_{A C}(t)=\left(\begin{array}{cccc}
\tilde{\rho}_{11}(t) & 0 & 0 & 0 \\
0 & \widetilde{\rho}_{2,2}(t) & 0 & \widetilde{\rho}_{2,4}(t) \\
0 & 0 & \tilde{\rho}_{3,3}(t) & 0 \\
0 & \tilde{\rho}_{2,4}^{*}(t) & 0 & \tilde{\rho}_{4,4}(t)
\end{array}\right),
$$

where $\tilde{\rho}_{3,3}(t)=\tilde{\rho}_{3,3}(0), \tilde{\rho}_{11}(t)=1-\sum_{i=2}^{4} \tilde{\rho}_{i i}(t)$ with

$$
\begin{aligned}
\tilde{\rho}_{22}(t)= & \frac{e^{-A_{+} t}}{\Omega_{1}^{2}+\Omega_{2}^{2}}\left\{\left(|\Delta|^{2}+\frac{\Omega_{2}^{2}+\Omega_{1}^{2}}{4}\right) \cosh \left(\Omega_{1} t\right)+\left(\frac{\Omega_{2}^{2}+\Omega_{1}^{2}}{4}\right.\right. \\
& \left.-|\Delta|^{2}\right) \cos \left(\Omega_{2} t\right)+\left(\frac{\Omega_{2} A_{-}}{2}-\frac{\Omega_{1} \eta}{2}\right) \sin \left(\Omega_{2} t\right)+\left(\frac{\Omega_{2}}{2} \eta\right. \\
& \left.\left.+\frac{\Omega_{1}}{2} A_{-}\right) \sinh \left(\Omega_{1} t\right)\right\},
\end{aligned}
$$

$$
\begin{gathered}
\tilde{\rho}_{44}(t)=\frac{\varepsilon_{\text {eff }}^{2}}{\Omega_{1}^{2}+\Omega_{2}^{2}}\left[\cosh \left(\Omega_{1} t\right)-\cos \left(\Omega_{2} t\right)\right] e^{-A_{+} t} \\
\tilde{\rho}_{2,4}(t)=\frac{e^{-A_{+} t}}{\Omega_{1}^{2}+\Omega_{2}^{2}}\left\{\frac{i \varepsilon_{\text {eff }}}{2}\left(\Omega_{2}-i \Omega_{1}\right)\left[\sin \left(\Omega_{2} t\right)+-i \sinh \left(\Omega_{1} t\right)\right]\right. \\
\left.+\Delta \varepsilon_{\text {eff }}\left[\cosh \left(\Omega_{1} t\right)-\cos \left(\Omega_{2} t\right)\right]\right\}
\end{gathered}
$$

where $A_{ \pm}=k \pm 2 \Gamma, 2 \Delta=\eta+i A_{-}$

$$
\Omega_{i}=\left[(-1)^{i} \frac{P}{2}+\frac{1}{2}\left(P^{2}-4 V\right)^{1 / 2}\right]^{1 / 2},
$$

with $P=\eta^{2}+4 \varepsilon_{e f f}^{2}-A_{-}^{2}$ and $V=-A_{-}^{2} \eta^{2}$ and $\omega=\omega_{0}$. We emphasize that, on the basis of the block diagonal form exhibited by $\widetilde{\rho}_{A C}(t)$, the transformed system, at a generic time instant $t$, is in a statistical mixture of the vacuum state $|0\rangle|-\rangle_{1}|-\rangle_{2}$ and of a one-excitation appropriate density matrix describing with certainty the storage of the initial energy. Taking into account the easily demonstrable inequality $\Omega_{1}<A_{+}$, it is immediate to convince oneself that for $t \gg A_{+}^{-1}$ the correspondent asymptotic form assumed by $\widetilde{\rho}_{A C}$ is time independent and such that the probability of finding energy in the effective JC subsystem exactly vanishes. Considering that the initial condition imposed on $\rho_{A C}(0)$ may be converted into $\widetilde{\rho}_{i i}$ $=\frac{1}{2}\left(\delta_{i 2}+\delta_{i 3}\right)$ and $\widetilde{\rho}_{i j}=0$ for $i \neq j$, we may conclude that at $t$ $\gg A_{+}^{-1}$ the only two matrix elements different from zero are $\widetilde{\rho}_{11}(t) \equiv \widetilde{\rho}_{22}(0)$ and $\widetilde{\rho}_{33}(t) \equiv \widetilde{\rho}_{33}(0)$. Transforming $\widetilde{\rho}_{A C}(t)$ back to the original representation, the exact solution $\rho_{A C}$ for the reduced density matrix of the system under scrutiny is then easily found. Since the unitary operator $U$ is time independent, we are legitimated to forecast an asymptotic timeindependent solution in the original representation too. The reduced density matrix can indeed be written in the compact form

$$
\rho_{A C}=\frac{1}{2}\left|\psi_{D}\right\rangle\left\langle\psi_{D}\left|+\frac{1}{2}\right| \psi_{T}\right\rangle\left\langle\psi_{T}\right|
$$

with $\left|\psi_{D}\right\rangle=|0\rangle|-\rangle_{1}|-\rangle_{2}$ and

$$
\left|\psi_{T}\right\rangle=\frac{1}{2}\left[|-\rangle_{1}|+\rangle_{2}-|+\rangle_{1}|-\rangle_{2}\right]
$$

eigenstate of $\left(\vec{\sigma}_{1}+\vec{\sigma}_{2}\right)^{2}$ with eigenvalue zero. Equation (21) suggests that stationary entangled states of the two atoms can be generated by putting them outside of the cavity single-photon detectors, allowing a continuous monitoring of the decay of the system through the two possible channels (atomic and cavity dissipation). Reading out the detectors states at $\bar{t} \gg A_{+}^{-1} \approx k^{-1}$, if no photon has been emitted, then as a consequence of this measurement outcome, our system is projected into the state $\left|\psi_{T}\right\rangle$ given by Eq. (22). This is the main result of our paper which means that a successful measurement, performed at large enough time instants, generates 
an uncorrelated state of the two subsystems atoms and cavity, leaving the atomic sample in its maximally antisymmetric entangled state (22). To analyze the time evolution of the degree of entanglement that gets established between the two initially uncorrelated atoms, we exploit the concept of concurrence $C$ first introduced by Wootters [26,27]. If at an assigned time $t$, no photons have been emitted, the conditional concurrence $C$ assumes the form

$$
C(t)=\frac{\left|\widetilde{\rho}_{22}(t)-\widetilde{\rho}_{33}(t)\right|}{\tilde{\rho}_{22}(t)+\widetilde{\rho}_{33(t)}+\widetilde{\rho}_{44}(t)} .
$$

As clearly shown in Fig. 1, the degree of entanglement between the two atoms, starting from zero, reaches its maximum value, that is $C=1$, in a time of the order of $k^{-1}$, after a large number of oscillations.

To summarize, in this paper we have analyzed the dynamics of a system composed of two two-level atoms with dipole-dipole interaction, embedded in a bad single-mode cavity and coupled to a common electromagnetic environment. The exact analytic solution of the Markovian dissipative dynamics reveals that the environment induces stationary entangled states of the two atoms, starting from a

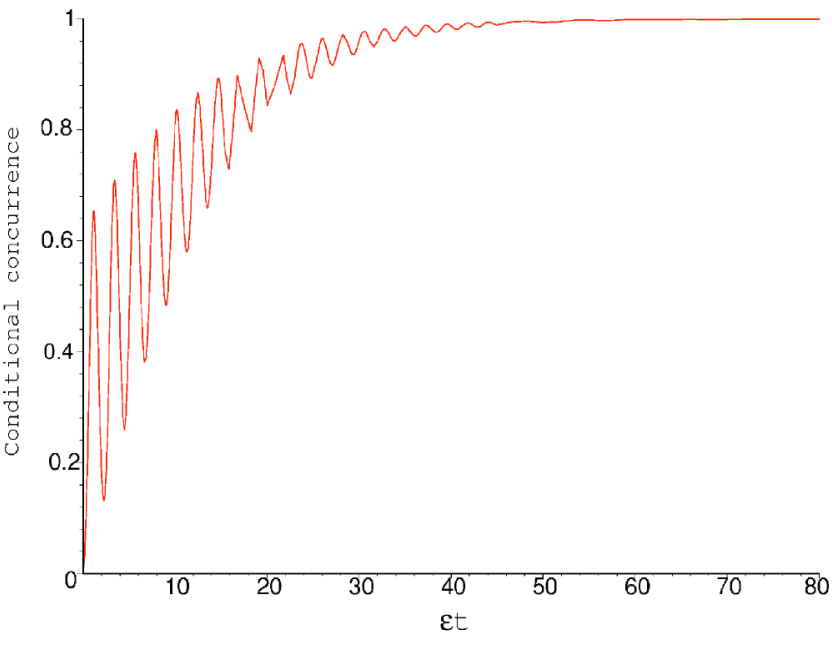

FIG. 1. Conditional concurrence $C$ in corresponding to $\eta$ $=0.5 \varepsilon, k=10^{-1} \varepsilon$, and $\Gamma=10^{-2} \varepsilon$.

realizable preparation of the matter subsystem. This fact make it possible the experimental realization of this process with real atoms and could be of some relevance for the development of quantum devices.
[1] M. A. Nielsen and I. L. Chuang, Quantum Computation and Quantum Information (Cambridge University Press, Cambridge, 2000).

[2] Guo Ping Guo et al., Phys. Rev. A 65, 042102 (2002); LuMing Duan et al., ibid. 57, 2399 (1998).

[3] J. I. Cirac and P. Zoller, Phys. Rev. A 50, R2799 (1993).

[4] S. Shneider and G. J. Milburn, Phys. Rev. A 65, 042107 (2002)

[5] G. J. Yang et al., Phys. Rev. A 59, 4012 (1998).

[6] E. Hagley et al., Phys. Rev. Lett. 79, 1 (1997).

[7] P. Foldi et al., Phys. Rev. A 65, 021802 (2002).

[8] Z. Ficek and R. Tanas, Phys. Rep. 372, 369 (2002).

[9] M. B. Plenio et al., Phys. Rev. A 59, 2468 (1999).

[10] T. Pellizzari et al., Phys. Rev. Lett. 75, 3788 (1995).

[11] F. Benatti et al., Phys. Rev. Lett. 91, 070402 (2003).

[12] A. Beige et al., Proc. SPIE 5111, 370 (2003).

[13] A. Beige, Phys. Rev. A 67, 020301(R) (2003).

[14] G. M. Palma et al., Proc. R. Soc. London, Ser. A 452, 567
(1996).

[15] Z. Ficek and R. Tanas, e-print quant-ph/0302124.

[16] L. Jakobczyk, J. Phys. A 35, 6383 (2002).

[17] F. Seminara and C. Leonardi, Phys. Rev. A 42, 5695 (1990).

[18] C. Leonardi et al., Riv. Nuovo Cimento 9, 1 (1986).

[19] E. T. Jaynes and F. W. Cummings, Proc. IEEE 51, 89 (1963).

[20] S. Haroche, in Fundamental Systems in Quantum Optics (North-Holland, Amsterdam, 1992), p. 767.

[21] H. P. Breuer and F. Petruccione, The Theory of Open Quantum Systems (Oxford University Press, Oxford, 2002).

[22] W. H. Louisell, Quantum Statistical Properties of Radiation (Wiley, New York, 1973).

[23] G. S. Agarwal, Quantum Optics, in Springer Tracts in Modern Physics (Springer-Verlag, Berlin, 1974), Vol. 70.

[24] G. Benivegna and A. Messina, Phys. Lett. A 126, 4 (1988).

[25] G. Benivegna et al., J. Mod. Opt. 36, 1205 (1989).

[26] S. Hill et al., Phys. Rev. Lett. 78, 5022 (1997).

[27] W. K. Wootters, Phys. Rev. Lett. 80, 2245 (1998). 